\title{
Supernova Remnants with Fermi Large Area Telescope
}

\author{
M. Caragiulo ${ }^{1,2, a}$ and L. Di Venere ${ }^{1,2}$ on behalf of Fermi-LAT Collaboration \\ ${ }^{1}$ Dipartimento Interateneo di Fisica "Michelangelo Merlin", Via Amendola 173, 70126 Bari, Italy \\ ${ }^{2}$ Istituto Nazionale di Fisica Nucleare (INFN), Sezione INFN di Bari, Via Orabona 4, 70126 Bari, Italy
}

\begin{abstract}
The Large Area Telescope (LAT), on-board the Fermi satellite, proved to be, after 8 years of data taking, an excellent instrument to detect and observe Supernova Remnants (SNRs) in a range of energies running from few hundred MeV up to few hundred $\mathrm{GeV}$. It provides essential information on physical processes that occur at the source, involving both accelerated leptons and hadrons, in order to understand the mechanisms responsible for the primary Cosmic Ray (CR) acceleration. We show the latest results in the observation of Galactic SNRs by Fermi-LAT.
\end{abstract}

\section{Introduction}

The LAT, the primary instrument on-board the Fermi Gamma-ray Space Telescope (Fermi), is an imaging, wide-field-of-view, pair-conversion telescope, which detects photons from few tens of $\mathrm{MeV}$ to few hundreds of GeV. It contains a high-resolution converter/tracker (composed of 16 planes of tungsten to promote the conversion of $\gamma$-rays into pairs, interleaved with silicon strip detectors) for direction measurement of the incident $\gamma$-rays, a $\mathrm{CsI}(\mathrm{Tl})$ crystal electromagnetic calorimeter for energy measurement of the incoming photon, and an anti-coincidence detector to identify the background of charged particles, mostly due to charged CRs [1].

Recently, the Fermi-LAT collaboration has released the latest version of Instrument Response Functions (IRFs), along with the reprocessed data, obtained with the new event reconstruction (tracker, calorimeter and ACD reconstruction), known as PASS 8. All these modifications contribute to improve the effective area, the angular and energy resolutions. Combined with the large amount of data collected by the LAT since its launch, PASS 8 is a powerful tool to study the emission below $100 \mathrm{MeV}$ and the extended sources, as it will be shown in section 3.2.

The Fermi-LAT is providing an unprecedented wealth of detections and observations of $\gamma$-ray sources. The detection of $\gamma$-rays is a clear signature that non-thermal processes are happening at the source, eventually accelerating particles. Since photons are not deflected by magnetic fields, they provide key information to study these phenomena.

\section{Supernova Remnants as sources of Cosmic Rays}

A Supernova Remnant (SNR) results from the interaction between the stellar material ejected by a Supernova (SN) explosion and the environmental gas. SNRs are considered the most probable

\footnotetext{
ae-mail: micaela.caragiulo@ba.infn.it
} 
sources of Galactic CRs, with energy from about $1 \mathrm{GeV}$ to about $10^{8} \mathrm{GeV}$ (SNR paradigm) [? ], and the acceleration processes take place at the SNR shocks.

The acceleration mechanism, also known as diffuse shock acceleration (DSA) theory, naturally predicts that the accelerated particles distribution is well described by a power law $\propto E^{-q}$ with a universal slope, whose spectral index in case of strong SNR shocks is $q=2$. A generalization of this theory, known as Non-Linear Diffusive Shock Acceleration (NLDSA) theory, takes into account the dynamical reaction of the accelerated particles on the shock, predicting a steeper particle distribution, compatible with the spectrum measured at Earth [2]. The CR flux observed at Earth $F(E)\left(\propto E^{-2.75}\right)$ is proportional to the injection spectrum at the source $Q_{i n j}(E)\left(\propto E^{-q}\right)$ and the Galactic permanence time $\tau_{\text {esc }}(E)\left(\propto E^{-\delta}\right)$, due to the propagation of CRs through the Galaxy:

$$
F(E)=Q_{i n j}(E) \tau_{e s c}(E) \propto E^{-(q+\delta)} .
$$

The model for CR propagation, based on the CR isotope and secondary-to-primary composition, provides a value of $\delta \sim 0.3-0.6$, suggesting a injection spectrum index $q=2.1-2.4$, compatible with the NLDSA predictions.

SNRs would be able to provide the required CR energetics, indeed the acceleration mechanism is efficient enough to account for the observed CR energy density $\rho_{C R} \sim 1 \mathrm{eV} / \mathrm{cm}^{3}$. $\rho_{C R}$ can be expressed as:

$$
\rho_{C R}=R_{S N} E_{S N} \tau_{e s c}(E) \epsilon,
$$

where $R_{S N} \sim 3 \mathrm{SN} /$ century is the SN explosion rate in our Galaxy, $E_{S N} \sim 10^{51} \mathrm{erg}$ is the typical explosion energy of a SN, $\tau_{e s c}(E) \sim 10^{7}$ years is the Galactic CR confinement time in the Galaxy and $\epsilon$ is the required acceleration efficiency, which has to be close to $\sim 10 \%$. Some models have already shown that NLDSA can provide such acceleration efficiencies [3].

An important evidence of the NLDSA is given by the observation of narrow filaments of nonthermal X-ray radiation along the shock front in young SNRs [4]. They are due to the synchrotron emission of accelerated electrons in a strong amplified magnetic field, which might be originated from the interaction of the charged accelerated particles with the shock.

\section{Gamma-ray observations}

The accelerated CRs interact with the SNR environment producing photons of energy range from radio to $\mathrm{TeV}$. The predominant processes in $\mathrm{MeV}-\mathrm{GeV}$ energy range are:

- Inverse Compton (IC) scattering of accelerated electrons on local photons from Cosmic Microwave Background and infrared radiation due to dust emission,

- bremsstrahlung radiation of the same electron population deflected in the Coulomb field, generated by charged particles of the gas surrounding the remnant,

- hadronic interaction between accelerated particles and this gas, which produces many hadrons, especially neutral pions, which then decay in photons.

The $\gamma$-ray spectrum due to the $\pi^{0}$-decay, with its peculiar shape due to the kinematic cut-off around $100 \mathrm{MeV}$ of the $\pi^{0}$ decay process, represents the hint of the so-called SNR paradigm for CRs since its production involves accelerated hadrons. For this reason, the observation of this kind of $\gamma$-ray emission from SNRs is essential to infer about the accelerations mechanisms. 


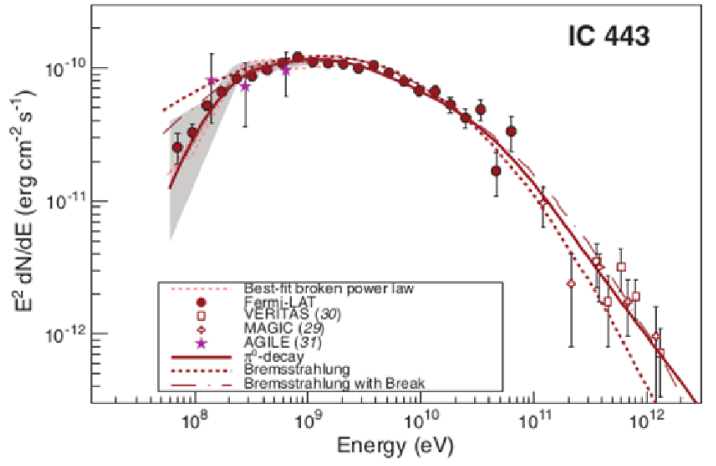

(a)

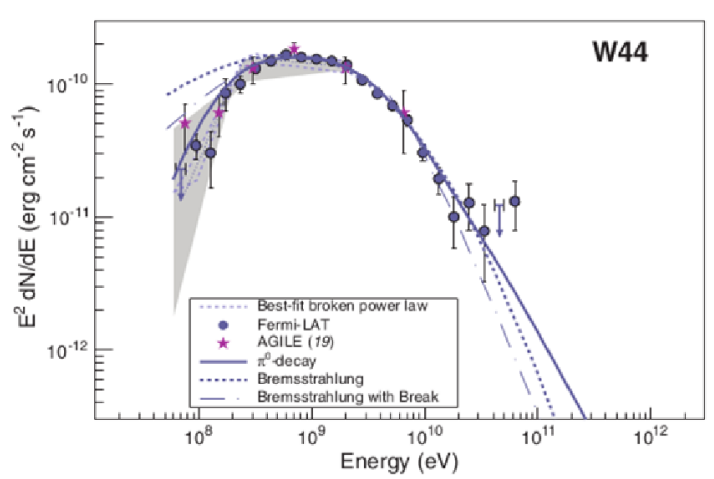

(b)

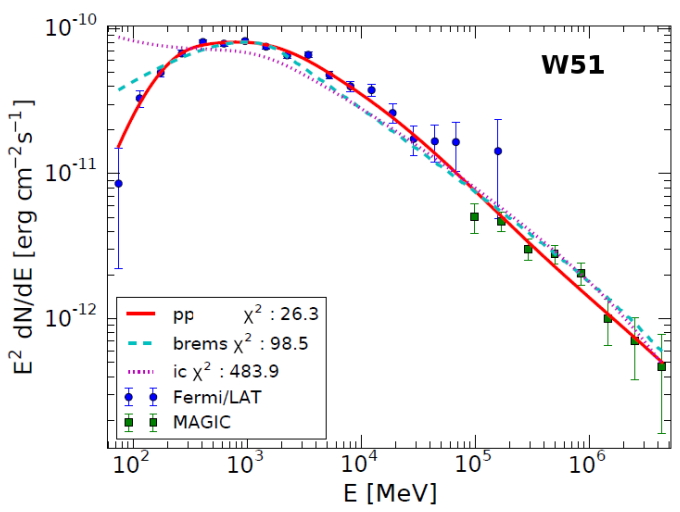

(c)

Figure 1: Spectral Energy Distribution of three SNRs, overlaid with theoretical models based either on a hadronic or leptonic interpretation of $\gamma$-ray emission. The shape of the observed $\gamma$-ray spectrum favors the hadronic interpretation. (a): IC443 [6]. (b): W44 [6]. (c): W51 [7].

\subsection{Fermi-LAT detection of hadronic interaction}

SNRs interacting with molecular clouds (MCs) are old objects in which the acceleration process is not very efficient anymore, as suggested by the steep $\gamma$-ray spectrum at high energies, which is probably due to the escaping of high energy CRs. Indeed, their strong $\gamma$-ray emission is not due to a high flux of relativistic hadrons, but to a very dense cloud target in the vicinity of the remnant, which interacts with the latter via nuclear collisions. Therefore, they do not give the best view of the CR acceleration but they are useful to investigate the CR propagation around sources and the escape from them.

The first detection of $\gamma$-ray spectrum due to the hadronic interaction has been found in the observation of tree middle aged SNRs interacting with MCs, few years ago W 44, IC 443 [5] [6] and recently W51 [7]. Figure 1 shows the Spectral Energy Distributions (SEDs) of these three SNRs. The data points at energies below $200 \mathrm{MeV}$ strongly support the hadronic interpretation. 


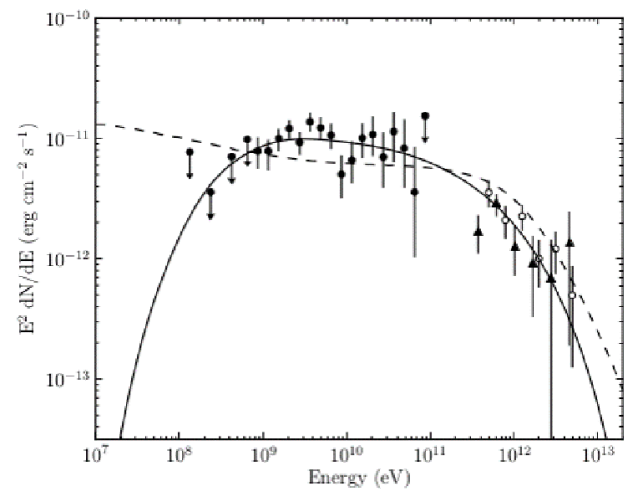

(a)

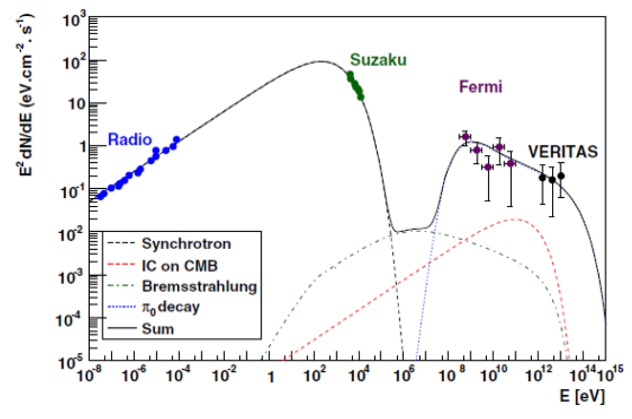

(b)

Figure 2: Spectral Energy Distribution of two young SNRs, overlaid with theoretical models. (a): Cassiopeia A. The lines represent two different interpretation models based on a hadronic (solid) or leptonic (dashed) origin of $\gamma$-ray emission [9]. (b): Tycho. The lines represent the contribution of different emission mechanisms. The total $\gamma$-ray emission (solid line) is dominated by the $\pi^{0}$-decay spectrum [8].

Young SNRs ( few thousand years old), instead, are at the initial stage of their evolution, so they are evolving in cleaner (and in most cases low-density) environments. For this reason, a detection of their $\gamma$-ray emission could give clear information about the acceleration processes taking place in these SNRs.

The best candidates as CR accelerators seem to be Tycho [8] and Cassiopeia A (Cas A) [9]. Their $\gamma$-ray spectrum detected by Fermi-LAT seems to be compatible with the hadronic model. In particular, the $\gamma$-ray flux of Cas A is characterized by a peculiar shape of the spectrum at energies below $100 \mathrm{MeV}$, where the $\pi^{0}$-decay spectrum presents a break due to the threshold energy of the pion production. On the other hand, other young SNRs, such as RX J1713.7-3946 [10] and RCW 86 [11], show different spectrum shapes (with a very hard spectral index in the $\mathrm{MeV}-\mathrm{GeV}$ energy range), which support the leptonic model, based on the production of $\gamma$-ray via IC scattering. The leptonic interpretation is also favored when considering the H.E.S.S. results [12] [13]. For these two SNRs, the absence of $\gamma$-rays from $\pi^{0}$-decay does not exclude the possibility of an efficient accelerated of CRs, but might be due to a low gas density around the source, which reduces the hadronic contribution to the photon flux. Figures 2 and 3 show the SEDs of these young SNRs with the best-fit interpretation models overlaid.

\subsection{Morphology study with PASS 8}

An important aspect of the study of $\gamma$-ray sources is the detection of their spatial shape, since it is an important characteristic for correctly associating $\gamma$-ray emitting sources with their counterparts at other wavelengths. This problem is complicated by the large PSF of the instrument. However, the improvement in PSF and effective area introduced by the PASS 8 reconstruction method has allowed the study of the spatial extension of some sources.

RCW 86 is one of the SNRs which have been recently detected as extended with PASS 8 . Figure 4 shows a test statistic map of this source observed by the LAT, compared with the contours of the same sources observed by the H.E.S.S. experiment [13]. In order to determine the best morphology of RCW 


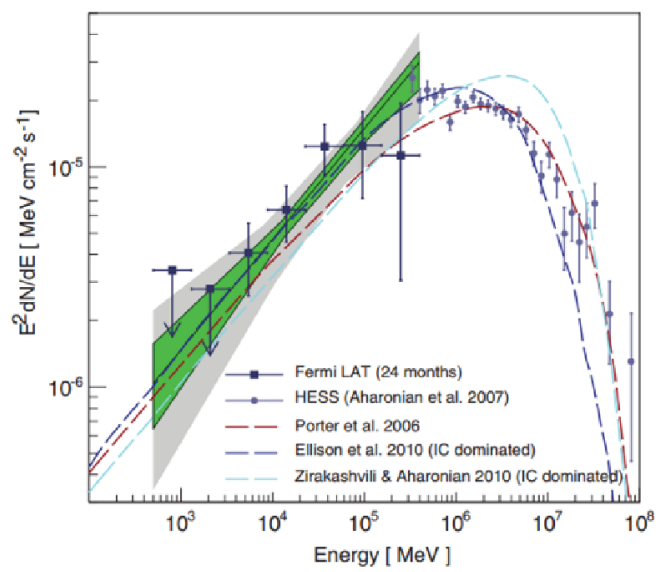

(a)

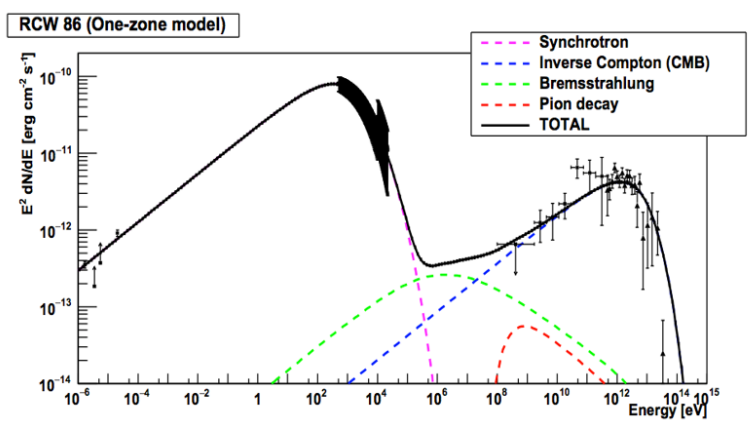

(b)

Figure 3: Spectral Energy Distribution of two young SNRs, overlaid with theoretical models based on a leptonic interpretation of the $\gamma$-ray emission. (a): RX J1713.7-3946. The lines represent different interpretation models in which the $\gamma$-ray emission (solid line) is dominated by the IC scattering [10]. (b): RCW 86. The lines represent the contribution of different emission mechanisms. The total $\gamma$-ray emission (solid line) is dominated by the IC scattering [11].

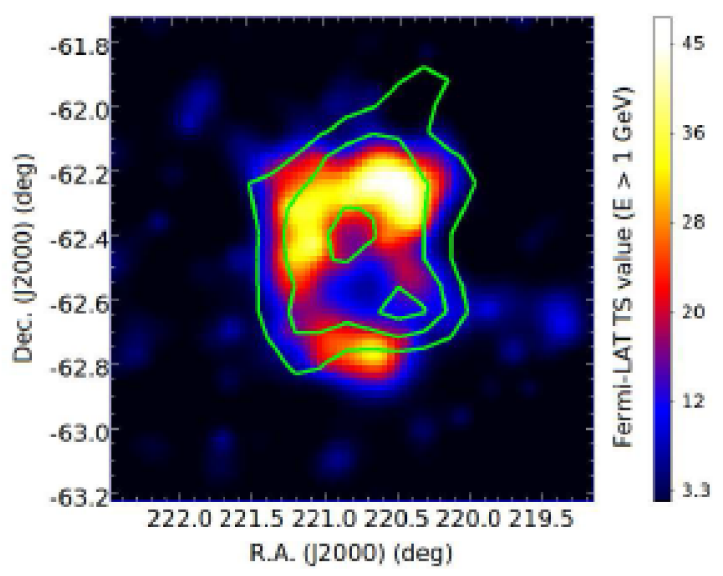

Figure 4: Test statistic maps of RCW 86 SNR seen by Fermi-LAT. The map is overlaid to the contours of the same source observed by H.E.S.S. experiment [11]. 


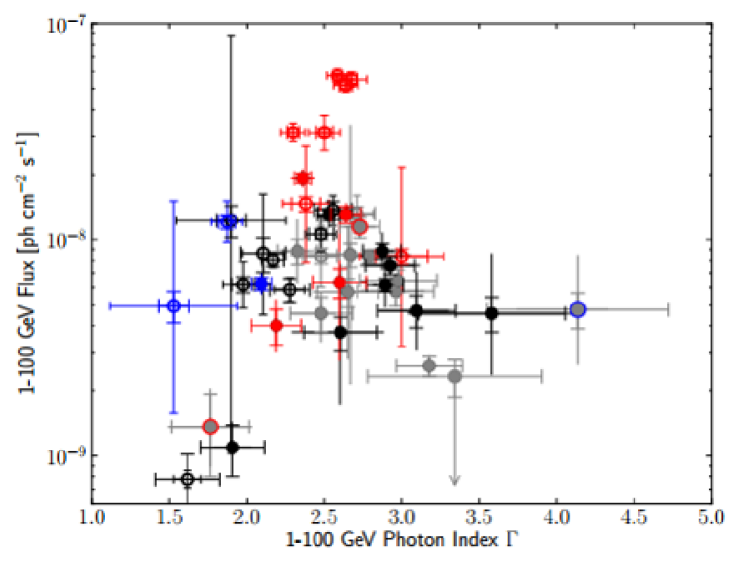

Figure 5: Scatter plot between $\gamma$-ray flux and spectral index of the SNRs detected in the first SNR catalog. Black: classified SNRs, grey: marginal candidates. Red: SNRs interacting with a molecular cloud, blue: young SNRs. Full circles: point-like sources, empty circles: extended sources [14].

86, the data have been fitted with different spatial models, simple or derived from measurements in other energy ranges [11]. The analysis revealed that the template provided by H.E.S.S., which detects $\gamma$-rays with energy above $\sim 1 \mathrm{TeV}$, is the one that better describes the LAT data. This supports the hypothesis that the $\gamma$-ray emission observed by the two experiments are due to the same particle population and the same emission mechanism. In this case, the whole SNR modeling favors the leptonic model, based on the IC scattering of accelerated electrons on the CMB photons, because of the very hard spectral index in the $\mathrm{MeV}-\mathrm{GeV}$ energy range.

\subsection{The $1^{\text {st }}$ Supernova Remnant Catalog}

A systematic study of an entire population of SNRs has been recently completed by the Fermi-LAT collaboration, producing the $1^{\text {st }}$ Supernova Remnant catalog [14]. Three years of data between $1 \mathrm{GeV}$ and $100 \mathrm{GeV}$ have been analyzed to study the SNRs already detected in other energy ranges (274 SNRs included in the catalog of Green [15], plus five additional SNRs identified after its publication), resulting in the observation of 36 SNRs, with the detection of 14 new sources. A detailed analysis of the localization and extension of the sources has been performed, as well as of the systematic errors due to the uncertainty on the instrument response and on the diffuse background modeling, which significantly affects the estimation of the source characteristics. A multi-wavelength study has also been done when possible, searching for example for possible correlations between $\gamma$-ray and radio or $\mathrm{X}$-ray spectral index. Figure 5 summarizes the results of the catalog, showing the scatter plot between the $\gamma$-ray flux and spectral index of the detected sources.

\section{Conclusions}

Fermi-LAT has an excellent capability to investigate the $\gamma$-ray spectrum of the astrophysical objects in the Galactic plane, such as SNRs. It is essential to understand the origin of these $\gamma$-ray emission, giving also information on non-thermal processes involving accelerated particles. Many SNRs have 
been detected and studied, with different characteristics, but a more systematic study is necessary to have a definite proof of CR acceleration in SNRs. For this purpose, the $1^{\text {st }}$ SNR catalog has been produced and future updates with more data and the new PASS 8 performances will provide more information.

Furthermore, the improved spatial resolution introduced with PASS 8 is allowing the identification and also more precise studies of the morphology of the brightest extended sources, giving the possibility to compare them with the templates observed at other wavelengths. New results have to be expected from Fermi-LAT in the next years.

\section{Acknowledgments}

The Fermi-LAT Collaboration acknowledges support for LAT development, operation and data analysis from NASA and DOE (United States), CEA/Irfu and IN2P3/CNRS (France), ASI and INFN (Italy), MEXT, KEK, and JAXA (Japan), and the K.A. Wallenberg Foundation, the Swedish Research Council and the National Space Board (Sweden). Science analysis support in the operations phase from INAF (Italy) and CNES (France) is also gratefully acknowledged.

\section{References}

[1] W.B. Atwood et al., Astrophys. J. 697, 1071 (2009), 0902 . 1089

[2] P. Blasi, Astron. Astrophys. Rev. 21, 70 (2013), 1311.7346

[3] G. Morlino, D. Caprioli, Astron. Astrophys. 538, A81 (2012), 1105.6342

[4] S. Katsuda et al., Astrophys. J. 709, 1387 (2010)

[5] A. Giuliani et al., Astrophys. J. Lett. 742, L30 (2011)

[6] M. Ackermann et al., Science 339, 807 (2013), 1302 . 3307

[7] T. Jogler, S. Funk, Astrophys. J. 816, 100 (2016)

[8] F. Giordano et al., Astrophys. J. Lett. 744, L2 (2012), 1108.0265

[9] Y. Yuan et al., Astrophys. J. 779, 117 (2013), 1310.8287

[10] A.A. Abdo et al., Astrophys. J. 734, 28 (2011), 1103.5727

[11] M. Ajello et al., Astrophys. J. 819, 98 (2016)

[12] F. Aharonian et al., Astron. Astrophys. 464, 235 (2007), astro-ph/0611813

[13] A. Abramowski et al., arXiv:1601.04461 (2016), 1601.04461

[14] F. Acero et al., Astrophys. J. Suppl. Series 224, 8 (2016), 1511.06778

[15] D.A. Green, Bulletin of the Astronomical Society of India 37, 45 (2009), 0905 . 3699 